\section{Commentary: Enhanced recovery program after congenital heart surgery: Promising baby steps}

\author{
Alexander Zhu, BS, Nicholas D. Andersen, MD, and \\ Veerajalandhar Allareddy, MD
}

Enhanced Recovery After Surgery (ERAS) programs have been praised as important measures to help expedite recovery, improve outcomes, and increase patient satisfaction. ${ }^{1}$ ERAS programs have yielded significant results in many surgical arenas, including abdominal surgery, cardiothoracic, and pediatric surgical populations, but have yet to be rigorously examined in the pediatric cardiothoracic surgical population. ${ }^{2-6}$ In the current issue of the Journal, Roy and colleagues ${ }^{7}$ report the preliminary outcomes of ERAS program use after congenital heart surgery in a targeted population with low comorbidities at a major children's hospital. Results showed reduced duration of mechanical ventilation, intensive care unit length of stay, and readmission within 30 days compared with pre-ERAS program patients. ${ }^{7}$ Further, there was no increase in reported complications or reinterventions. However, the change in overall postoperative hospital length of stay was not significant.

While these findings represent an encouraging first step in the development of an ERAS program in congenital cardiac surgery, a few factors limit the strength of the results. The study was a retrospective, nonrandomized study observing the first 5 months of program implementation. However, to minimize observational bias, a robust propensity scoring analysis was performed. Adherence with several ERAS program protocols was suboptimal, including parameters such as ingestion of glucosecontaining beverage before surgery $(17 \%)$, hypoglycemia during surgery $(17 \%)$, postoperative nausea and vomiting prevention $(33 \%)$, and mechanical ventilation less than 8 hours $(54 \%)$, as well as many others. ${ }^{7}$ Nevertheless, the results obtained seem congruent with previous studies of

From Duke University Medical Center, Durham, NC.

Disclosures: Authors have nothing to disclose with regard to commercial support.

Received for publication Nov 2, 2019; accepted for publication Nov 3, 2019; available ahead of print Nov 27, 2019

Address for reprints: Nicholas D. Andersen, MD, Division of Cardiovascular and Thoracic Surgery, Duke University Medical Center, 2301 Erwin Rd, DUMC 3474, Durham, NC 27710 (E-mail: Nicholas.Andersen@Duke.edu).

J Thorac Cardiovasc Surg 2020;160:1322-3

$0022-5223 / \$ 36.00$

Copyright (c) 2019 by The American Association for Thoracic Surgery

http://dx.doi.org/10.1016/j.jtcvs.2019.11.022

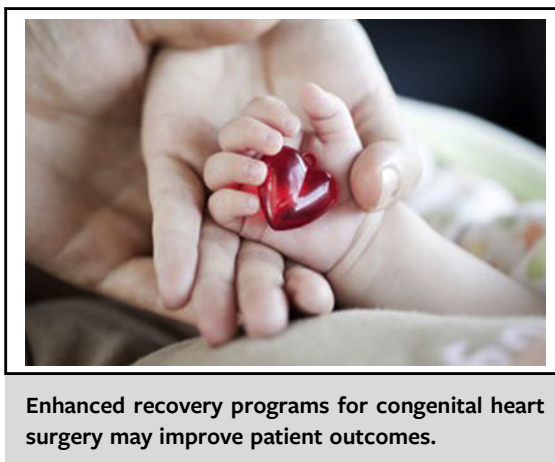

CENTRAL MESSAGE

A novel enhanced recovery program for congenital heart surgery shows promising results in its initial implementation.

ERAS implementation in the pediatric and cardiothoracic surgical populations. ${ }^{3-6}$

Through this early experience, the authors recognize there is much to improve. As stated previously, despite using this rigorous program, there was not optimal adherence and use. It is difficult to unify a multidisciplinary team as complex as the team caring for congenital heart surgery patients through an ERAS program. We foresee the more difficult barriers to achieving high compliance to this program are educating both provider and patients of the guidelines, especially at a large institution that may have many staff members. In addition, family dynamics may be another wild card in compliance toward the program.

Despite these limitations, strengths of this study are manyfold. The authors used a patient population with few comorbidities that was a good fit for the ERAS program. Moreover, an ERAS program created and supported by a multidisciplinary group of health care workers is fairly difficult. To achieve some adherence is impressive, and even more impressive are early results showing a benefit due to the program.

The main takeaway from this study is the framework that can serve as an inspiration for other children's hospitals across the nation. This ERAS program, in its infancy, shows encouraging results in how decreased variability in care, efforts to standardize care, and elimination of stylistic variables between health care providers provides more benefit to the patient-including optimization of resource use. Further, such a multidisciplinary collaborative approach has the potential to augment the overall family experience and improve patient satisfaction (Figure 1). This is promising work and a (baby) step in the right direction. 


\section{References}

1. Ljungqvist $\mathrm{O}$, Scott M, Fearon KC. Enhanced recovery after surgery: a review. JAMA Surg. 2017;152:292-8.

2. Thiele RH, Rea KM, Turrentine FE, Friel CM, Hassinger TE, McMurry TL, et al. Standardization of care: impact of an enhanced recovery protocol on length of stay, complications and direct costs after colorectal surgery. J Am Coll Surg. 2015;220: 430-43.

3. Rove KO, Edney JC, Brockel MA. Enhanced recovery after surgery in children: promising, evidence- based multidisciplinary care. Paediatr Anaesth. 2018;28: 482-92.
4. Shinnick JK, Short HL, Heiss KF, Santore MT, Blakely ML, Raval MV. Enhancing recovery in pediatric surgery: a review of the literature. J Surg Res. 2016;202:165-76.

5. Williams JB, McConnell G, Allender JE, Woltz P, Kane K, Smith PK, et al. Oneyear results from the first US-based enhanced recovery after cardiac surgery (ERAS Cardiac) program. J Thorac Cardiovasc Surg. 2019;157:1881-8.

6. Brown JK, Singh K, Dumitru R, Chan E, Kim MP. The benefits of enhanced recovery after surgery programs and their application in cardiothoracic surgery. Methodist Debakey Cardiovasc J. 2018;14:77-88.

7. Roy N, Fernanda Parra M, Brown ML, Sleeper LA, Nathan M, Sefton BA, et al. Initial experience introducing an enhanced recovery program in congenital cardiac surgery. J Thorac Cardiovasc Surg. 2020;160:1313-21.e5.
See Article page 1313.

\section{Commentary: The art of medicine versus paint by numbers}

\author{
Jennifer C. Romano, MD, MS
}

Medicine is unique from the rest of the scientific world, as there is an element of "art" to how it is practiced. We know how various organ systems are supposed to work, but when things go awry, the interpretation and treatment can be variable. This is where many find the challenge and joy of practicing medicine. However, it is this variability that has been the focus in quality improvement initiatives. Practice pattern variation has become synonymous with nonidealized outcomes. It is true, when organ systems or recovery pathways follow relatively consistent sequences, employing methods to minimize variation can improve outcomes and/or efficiencies. One could argue that creating guidelines and protocols takes away the "art" of caring for patients. The counter argument is that regimenting much of the care with known predictable pathways allows the physician more time and mental space to focus on the complex and challenging clinical scenarios in need of "artistic" consideration.

The institution, more so than the outcomes, of an Enhanced Recovery After Surgery program at Boston

\footnotetext{
From the Section of Congenital Heart Surgery, Department of Cardiac Surgery, University of Michigan, C. S. Mott Children's Hospital, Ann Arbor, Mich.

Disclosures: Author has nothing to disclose with regard to commercial support.

Received for publication Nov 12, 2019; accepted for publication Nov 12, 2019; available ahead of print Dec 9, 2019.

Address for reprints: Jennifer C. Romano, MD, MS, 11-733 C. S. Mott Children's Hospital, 1540 E. Hospital Dr, SPC 4204, Ann Arbor, MI 48109-4204 (E-mail: jhirsch@umich.edu).

J Thorac Cardiovasc Surg 2020;160:1323-4

$0022-5223 / \$ 36.00$

Copyright (c) 2019 by The American Association for Thoracic Surgery

http://dx.doi.org/10.1016/j.jtcvs.2019.11.074
}

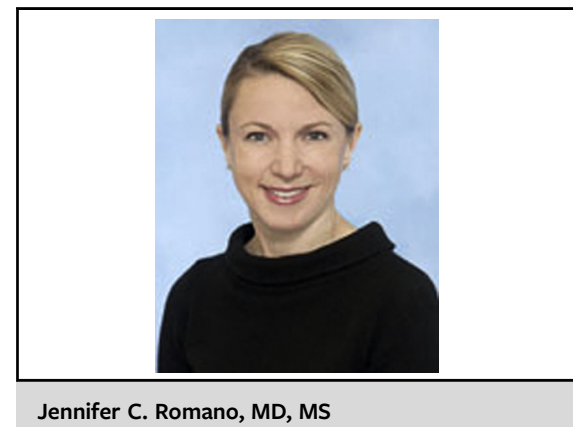

CENTRAL MESSAGE

Guidelines are used in the inpa-

tient setting to optimize effi-

ciency and quality of care. Some

say they restrict the "art" of

medicine. Implementing these

Children's "paint-by-number" guidelines can

Hospital is the be just as clinically challenging. focus of Roy and colleague's analysis. ${ }^{1}$ The findings are most notable for the Herculean effort to derive and implement a protocol-driven program to reduce length of stay and adverse outcomes while also improving patient and family satisfaction. The significant, albeit not clinically relevant, reduction in mechanical ventilation time and intensive care length of stay are the "outcomes" but should not be the focus. The real importance of this study is the demonstration of an incredibly robust multidisciplinary effort to outline a comprehensive perioperative plan to improve early extubation and feeding while minimizing opioids and nausea. I am impressed with the power of getting all disciplines and level of care providers in the same room to focus on one problem. It is striking how much can be learned as well as the enhanced commitment 